\title{
Calculation of the Impulse Response of Step-Index Plastic Optical Fibers Using the Time-Dependent Power Flow Equation
}

\author{
B. DrluaČA ${ }^{a}$, S. SAVOViĆ $\tilde{S}^{a, b, *}$ AND A. DJORDJEVICH ${ }^{b}$ \\ ${ }^{a}$ Faculty of Science, R. Domanovica 12, 34000 Kragujevac, Serbia \\ ${ }^{b}$ City University of Hong Kong, 83 Tat Chee Avenue, Hong Kong, China
}

\begin{abstract}
The analytical solution of the time-dependent power flow equation is employed to calculate impulse response in a step-index plastic optical fiber. Results are given at different fiber lengths and are shown to agree with those reported in the literature. Mode-dependent attenuation, modal dispersion and mode coupling in plastic optical fibers are known to affect fiber-optic power delivery, data transmission, and sensing systems.
\end{abstract}

PACS numbers: 42.81.-i, 42.81.Qb, 42.81.Uv

\section{Introduction}

Transmission properties of step-index (SI) multimode optical fibers depend strongly upon mode-dependent attenuation, modal dispersion and the rate of mode coupling (power transfer from lower to higher order modes) caused by intrinsic perturbation effects (primarily due to microscopic bends, irregularity of the core-cladding boundary and refractive index distribution fluctuations). Different simulation models are usually required for these three important effects in SI optical fibers. The ray tracing model calculates the trajectory for each ray through the fiber. It enables the calculation of the impulse response including the process of mode-dependent attenuation and modal dispersion. This model is computationally intensive as a large number of ray-trajectories must be generated. In contrast, using the time-independent power flow equation [1], mode-dependent attenuation and mode coupling can be modeled effectively to reveal their influence on transmission characteristics of the fiber. Furthermore, using the time-dependent power flow equation, one can model all three major fiber effects [2]. Using the time-dependent power flow equation, we report in this work on the impulse response in SI plastic optical fiber (POF) that has been investigated by Breyer et al. [3].

\section{Time-dependent power flow equation}

We use Gloge's time-dependent power flow equation to describe the evolution of the modal power distribution

\footnotetext{
* corresponding author; e-mail: savovic@kg.ac.rs
}

along the axis of the SI POF (as coordinate $z$ ). Individual modes are characterized by their inner propagation angle $\theta$ measured with respect to fiber axis. Gloge's time-dependent power flow equation can be written as [2]:

$$
\begin{aligned}
& \frac{\partial P(\theta, z, t)}{\partial z}+\frac{\partial t}{\partial z} \frac{\partial P(\theta, z, t)}{\partial t}=-\alpha(\theta) P(\theta, z, t) \\
& +\frac{1}{\theta} \frac{\partial}{\partial \theta}\left[\theta D(\theta) \frac{\partial P(\theta, z, t)}{\partial \theta}\right],
\end{aligned}
$$

where $t$ is time, $P(\theta, z, t)$ is power distribution over angle, space and time, $\alpha(\theta)$ is mode-dependent attenuation, $\partial t / \partial z$ is mode delay per unit length and $D(\theta)$ is mode-dependent coupling coefficient. Mode-dependent attenuation can be written in the form $\alpha(\theta)=\alpha_{0}+A \theta^{2}+\ldots$, where $\alpha_{0}$ describes the loss common to all modes. It can be accounted for later by multiplying the end-solution by the term $\mathrm{e}^{-\alpha_{0} z}[4]$. Therefore, in solving (1) one should consider only the term $A \theta^{2}$, which is most important among the higher order modes [1]. Assuming also that coupling coefficient $D$ is mode-independent, Eq. (1) can be written as follows [2]:

$$
\begin{aligned}
& \frac{\partial P(\theta, z, t)}{\partial z}+\frac{\partial t}{\partial z} \frac{\partial P(\theta, z, t)}{\partial t}=-A \theta^{2} P(\theta, z, t) \\
& +\frac{D}{\theta} \frac{\partial}{\partial \theta}\left[\theta \frac{\partial P(\theta, z, t)}{\partial \theta}\right] .
\end{aligned}
$$

The derivative $\partial t / \partial z$ can be obtained using the group velocity of a mode with characteristic angle $\theta$, which is

$$
\frac{\mathrm{d} z}{\mathrm{~d} t}=\frac{c}{n\left(1+\theta^{2} / 2\right)} \text {. }
$$

Neglecting the delay $n / c$ common to all modes, Eq. (2) could be rewritten [2], and after applying the Laplace transform 


$$
p(\theta, z, s)=\int_{0}^{\infty} \mathrm{e}^{-s t} P(\theta, z, t) \mathrm{d} t,
$$

Eq. (2) agrees with the time-independent power flow equation

$$
\frac{\partial p}{\partial z}=-A \sigma^{2} \theta^{2} p+\frac{D}{\theta} \frac{\partial}{\partial \theta}\left(\theta \frac{\partial p}{\partial \theta}\right)
$$

except for the factor $\sigma^{2}[1]$. One can therefore use the solution of the time-independent power flow equation, if $A$ is replaced by $A \sigma^{2}$. For the Gaussian input distribution one obtains [1]:

$$
p(\theta, z, s)=f(z, s) \exp \left(-\theta^{2} / \Theta^{2}(z, s)\right),
$$

where $\Theta^{2}(z, s)$ and $f(z, s)$ are described by Gloge [1].

For continuous wave excitation $(s=0)$, the angular width $\Theta(z, 0)$ changes monotonically from $\Theta_{0}$ to $\Theta_{\infty}$ as $z$ increases. Since the width $\Theta_{\infty}$ characterizes a distribution which propagates unchanged (at steady state) and with the minimum overall loss coefficient $\gamma_{\infty}$, it seems practical to excite this distribution right from the beginning $\left(\Theta_{0}=\Theta_{\infty}\right)[2]$. The closed-form Laplace transform of (6) exists only for the approximation given in the limits $z \ll 1 / \gamma_{\infty}$ (short fiber) and $z \gg 1 / \gamma_{\infty}$ (long fiber) [2].

In the case of short fiber, Eq. (6) becomes [2]:

$$
p(\theta, z, s)=\frac{f(0, s)}{1+\gamma_{\infty} z} \exp \left(-\theta^{2}\left(\frac{1}{\Theta_{0}^{2}}+\frac{n z}{2 c} s\right)\right) .
$$

The total output is obtained from the integration $p(\theta, z, s)$ over all angles [2]. With reference to (7) and for $z \ll 1 / \gamma_{\infty}$, one obtains [2]:

$$
q=\frac{\pi f(0, s) \Theta_{0}^{2}}{\left(1+\gamma_{\infty} z\right)\left(1+n \Theta_{0}^{2} z s / 2 c\right)} .
$$

If one sets $f(0, s)=1$, which corresponds to an infinitesimally short input pulse of energy 1 , the Laplace transformation of (8) yields the impulse response of the fiber [2]:

$$
Q(z, t)=\frac{2 c \pi}{n z\left(1+\gamma_{\infty} z\right)} \exp \left(-2 c t / n \Theta_{0}^{2} z\right),
$$

where $Q(t)$ is limited practically to a time interval narrower than $n \theta_{\mathrm{c}}^{2} z /(2 c)$, which is the delay between the fastest and the slowest mode $\left(\theta_{\mathrm{c}}\right.$ is the fiber's critical angle).

In case of a long fiber, Eq. (6) assumes the form [2]:

$$
p=\frac{2 \sigma}{1+\sigma} \exp \left(-\sigma\left(\theta^{2} / \Theta_{0}^{2}+\gamma_{\infty} z\right)\right) \text {, }
$$

which leads to

$$
q=\frac{2 \pi \Theta_{0}^{2}}{1+\sigma} \exp \left(-\sigma \gamma_{\infty} z\right)
$$

where $p$ is integrated over all angles $\theta$, as before. Introducing $\sigma=\sqrt{1+n s / 2 c A}$ into (12), one can form the Laplace transform of $q(s)$. By using the condition $\gamma_{\infty} z>1$, the impulse response of the fiber is

$$
\begin{aligned}
& Q(z, t)=\Theta_{0}^{2} \sqrt{\frac{\pi}{T t}}\left(\frac{t}{\gamma_{\infty} z T}+\frac{1}{2}\right)^{-1} \\
& \quad \times \exp \left(-\frac{\gamma_{\infty}^{2} z^{2} T}{4 t}-\frac{t}{T}\right)
\end{aligned}
$$

where

$$
T=\frac{n}{2 c A}=\frac{n}{2 c} \frac{\Theta_{0}^{2}}{\gamma_{\infty}}
$$

Besides the analytical solution of the time-dependent power flow Eq. (2) which is obtained by Gloge [2], two numerical approaches for solving the time-dependent power flow Eq. (1) have been reported recently: implicit finite-difference method (Crank-Nicholson scheme) by Breyer et al. [3] and explicit finite-difference method in the matrix form by Mateo et al. [5]. In this work using Gloge's analytical solution of (2), we calculate the impulse response of the SI POF investigated earlier by Breyer et al. [3] and compare our analytical results with their numerical results.

\section{Results}

Using Gloge's analytical solution of the time-dependent power flow Eq. (2) we have calculated impulse response of SI POF investigated earlier by Breyer et al. [3]. The fiber analyzed is the PREMIER GH4001 (GH fiber) from Mitsubishi with the diameter of $1 \mathrm{~mm}$ and numerical aperture $\mathrm{NA}=0.5$ (corresponding to inner critical angle of $\left.\theta_{\mathrm{c}}=19.5^{\circ}\right)$ and $0.15 \mathrm{~dB} / \mathrm{m}$ of nominal attenuation. In obtaining the impulse response of $\mathrm{GH}$ fiber, Breyer et al. [3] have assumed mode-dependent attenuation $\alpha(\theta)$ and mode-dependent coupling coefficient $D(\theta)$. In contrast, Gloge's analytical solution of the time-dependent power flow Eq. (2) is obtained assuming a constant coupling coefficient $D$ and constant $A$ ( $A$ is the second order multiplicative factor in the series expansion of the mode-dependent attenuation $\alpha(\theta)$ ). In order to obtain the value for $A$ for the fiber analyzed, we used the graph for $\alpha(\theta)$ in Fig. 1 proposed earlier by Mateo

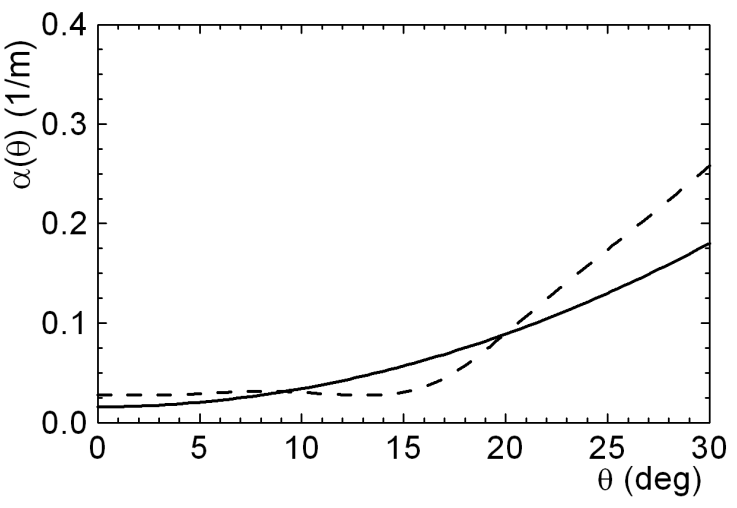

Fig. 1. Mode-dependent attenuation $\alpha(\theta)$ for GH fiber proposed by Mateo et al. [6] (dashed line) and our fit obtained using the function $\alpha(\theta) \approx \alpha_{0}+A \theta^{2}$, with $\alpha_{0}=$ $0.01591 / \mathrm{m}$ and $A=0.4025\left(\mathrm{rad}^{2} \mathrm{~m}\right)^{-1}$ (solid line).

et al. [6] and used in numerical calculations by Breyer et al. [3]. Since mode-dependent attenuation can be written in the form $\alpha(\theta) \approx \alpha_{0}+A \theta^{2}$, one can determine $A$ by fitting the graph shown in Fig. 1 using this function. In this way, we have obtained $\alpha_{0}=0.01591 / \mathrm{m}$ and $A=0.4025\left(\mathrm{rad}^{2} \mathrm{~m}\right)^{-1}$. The value of the constant 
coupling coefficient $D=1.171 \times 10^{-4} \mathrm{rad}^{2} / \mathrm{m}$ has been used in the first approach to the modeling of mode coupling in the analyzed fiber by Mateo et al. [6], which we have adopted in this work. One should mention here that modeling the mode coupling process with a constant $D$ is commonly done by many other authors $[1,4,7,8]$.

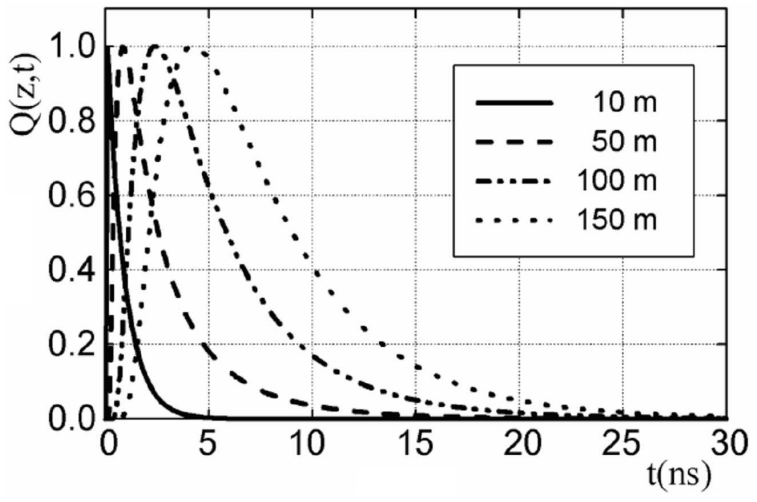

Fig. 2. Analytical results for the impulse response for different fiber lengths for GH fiber (this work).

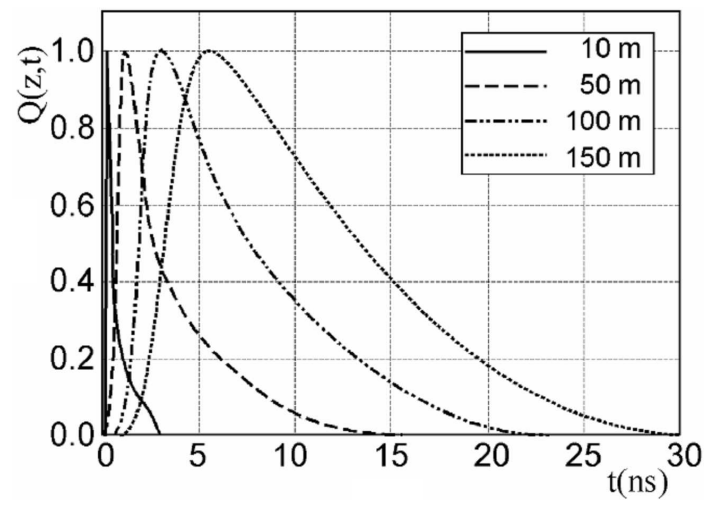

Fig. 3. Numerical results for the impulse response for different fiber lengths for GH fiber obtained by Breyer et al. [3] (retraced).

A Dirac impulse in time and a mode distribution with the width at $1 / \mathrm{e}$ of $\Theta_{0}=\Theta_{\infty}=9.85^{\circ}\left(\mathrm{FWHM}=8.35^{\circ}\right)$ at the beginning of the fiber is used. Equation (9), valid for short fiber lengths $z \ll 1 / \gamma_{\infty}=41.3 \mathrm{~m}$, has been used for calculating impulse response at $z=10 \mathrm{~m}$. Equation (12), valid in a case of long fiber lengths $z \gg 1 / \gamma_{\infty}$, has been used for calculating impulse response at fiber lengths $z=50,100$ and $150 \mathrm{~m}$. Our analytical results for the impulse response of the fiber analyzed are shown in Fig. 2. In Fig. 3, numerical results obtained by Breyer et al. [3] for impulse response are shown for the same fiber lengths. A good agreement between these results is apparent. One can observe that, at short fiber lengths $(z=10 \mathrm{~m})$, the mode-dependent attenuation is the dominant effect. With increasing fiber length, mode coupling begins to influence the impulse shape changing it to Gaussian.

Finally, the power exiting the fiber at the highest angles (tails of the Gaussian launch beam) has the longest delays. This suggests an efficient means of improving the fiber capability by spatial filtering-out of the tail at higher angles [5]. As most power is confined within the range of lower angles, such filtering-out of the power at the highest angles will cause only a small power loss while producing a narrower overall impulse response.

\section{Conclusion}

The analytical solution of the time-dependent power flow equation is employed to calculate the impulse response in a step-index plastic optical fiber that was investigated earlier by Breyer et al. [3]. Our results for the impulse response at different fiber lengths agree well with the earlier reported numerical results [3]. It has been observed that at short fiber lengths mode-dependent attenuation is the dominant effect. With the increasing fiber length, mode coupling begins to influence impulse response of the fiber, resulting in the impulse shape changing to a Gaussian-like shape. In general, using the information provided by the space-time power distribution, fiber transmission characteristics can be enhanced using an appropriate spatial filter. In doing so, a good fiber characterization can be applied to optimize the fiber performance in POF links.

\section{Acknowledgments}

The work described in this paper was supported by a grant from City University of Hong Kong (project No. 7002313) and by a grant from Serbian Ministry of Science (project No. 141037).

\section{References}

[1] D. Gloge, Bell Syst. Tech. J. 51, 1767 (1972).

[2] D. Gloge, Bell Syst. Tech. J. 52, 801 (1973).

[3] F. Breyer, N. Hanik, J. Lee, S. Randel, Proc. POF Modelling Workshop, Nurenberg (Germany), 2007, Eds. C.-A. Bunge, H. Poisel, 2007, p. 111.

[4] A. Djordjevich, S. Savović, J. Opt. Soc. Am. B 21, 1437 (2004).

[5] J. Mateo, M.A. Losada, J. Zubía, Opt. Express 17, 2850 (2009).

[6] J. Mateo, M.A. Losada, I. Garcés, J. Zubía, Opt. Express $\mathbf{1 4}, 9028$ (2006).

[7] W.A. Gambling, D.P. Payne, H. Matsumura, Appl. Opt. 14, 1538 (1975).

[8] G. Jiang, R.F. Shi, A.F. Garito, IEEE Photon. Technol. Lett. 9, 1128 (1997). 